3

\title{
Three-dimensional integral imaging display system via off-axially distributed image sensing
}

\author{
Yongri Piao ${ }^{1}$, Hongjia $\mathrm{Qu}^{1}$, Miao Zhang ${ }^{2}$, Myungjin $\mathrm{Cho}^{3}$ \\ ${ }^{1}$ School of Information and Communication Engineering, Dalian University of Technology, Dalian, 116024, China \\ ${ }^{2}$ School of Software Technology, Dalian University of Technology, Dalian, 116024, China \\ ${ }^{3}$ Dept. of Electrical, Electronic, and Control Engineering, IITC, Hankyong National University, Anseong, 456-749, South Korea \\ *Corresponding author: mjcho@hknu.ac.kr
}

Abstract

In this paper, we propose a three-dimensional integral imaging display system with a multiple recorded images using off-axially distributed image sensing. First, the depth map of the 3D objects is extracted from the offaxially recorded multi-perspective $2 \mathrm{D}$ images by using profilometry technique. Then, the elemental image array is computationally synthesized using the extracted depth map based on ray mapping model. Finally, the 3D images are optically displayed in integral imaging system. To show the feasibility of the proposed method, the optical experiments for 3D objects are carried out and presented in this paper.

Keywords: Off-axially distributed images sensing, Depth extraction, Elemental image array, 3D integral imaging 


\section{Introduction}

Integral imaging has been actively researched as one of the next-generation 3D display techniques [1-2], since it can be broadly applied to many fields including computer vision [3-5], image encryption [6-7], watermarking [8-9], etc. Within the group of auto-stereoscopic displays, integral imaging has glamorous and fascinating features of working with incoherent light, dealing with relatively small amount of data, having simple imaging architectures, and displaying full-color images with full parallax and continuous viewing points. Integral imaging consists of two processes: pickup and display. In the pickup process, rays coming from a 3D scene are recorded through a lenslet array by use of charge-couple device (CCD). The small images recoded on the CCD are regarded as elemental images, each of which has a difference perspective. On the other hand, in the display process, the 3D images are formed by integrating the rays from the elemental images by use of the similar lenslet array.

One of the main challenges in integral imaging is its low resolution of the reconstructed images. Many achievements have been accomplished in terms of this issue [10-15]. Among them, offaxially distributed image sensing (ODIS) [10-12] can record a 3D scene with both lateral and longitudinal perspectives, compared with axially distributed image sensing (ADS) [13-15]. In ODIS, the elemental images are recorded by a single camera translated along a slanted axis. In the display processing, the first attempts was to use a corresponding computational reconstruction algorithm for image display. It is worth to notice that lenslet arrays are not used either in the pickup or in the display process. Despite the fact that a lenslet array is not involved to capture a 3D scene in the ODIS system, elemental images still maintain lateral perspectives as in the integral imaging system, and meanwhile they have the additional longitudinal perspectives.

In this paper, we propose a 3D display system based on the ODIS architecture, in which elemental images with lateral and longitudinal perspectives. Due to the additional longitudinal information contained within the elemental images, the depth and intensity information of the 3D scene can be extracted based on use of statistical variance. With the extracted depth map and color 
image, the elemental images are computationally synthesized based on ray mapping model between 3D space and elemental image plane. As a result, 3D images can be optically reconstructed by integrating the rays from the synthesized elemental images through the lenslet array. To confirm the feasibility of the proposed method, optical and computational experiments are carried out and presented.

\section{3D integral imaging display using ODIS}

Figure 1 shows the block diagram of 3D integral imaging display with the off-axially recorded elemental images. It consists four steps: ODIS pickup process, profilometry-based depth extraction process, computationally elemental image array synthesis process, and 3D integral imaging display process.

\subsection{Off-axially recorded multi-perspective images}

A set of multi-perspective images are recorded by a single camera translated along the slanted axis $d$, as shown in Fig.2.. Suppose the focal length of the camera is $g$, the optical axis is $\mathrm{z}$, and $\theta$ denotes the angle between the slanted axis and the optical axis of the camera. $N$ images can be recorded by shifting the camera along the slanted axis with $N-1$ times. The separation distance between two adjacent cameras is $\Delta d$ as depicted in Fig. 2. The $i$-th camera position can be calculated by from the first camera $d_{i}=d_{1}+(i-1) \times \Delta d$, where $d_{1}$ is the distance between the origin $(z=0, x=0)$ and the first image sensor. Due to the face that each recorded 2D image has a different magnification ratio and offset, i.e., the $2 \mathrm{D}$ image with the smallest magnification ratio can be obtained when $i=1$ because the camera position is farthest from 3D object, this magnification ratio difference between recorded $2 \mathrm{D}$ images is useful to estimate and extract depth information of 3D objects.

\subsection{Profilometry-based depth extraction}

As mentioned in [16], the depth information can be extracted by using the profilometry for the off-axially recorded multi-perspective image set. Image points in different images 
corresponding to a 3D object point are located at different positions according to the camera position as shown in Fig. 3. All intensities of image points may be the same or similar when we reconstruct the integrated rays at the original position of 3D object point. Otherwise, when the estimation position is not equal to the original position of $3 \mathrm{D}$ object point, reconstructed image points will have different intensities. Based on this principle, depth information of 3D objects can be estimated by using the statistical variance of intensity distribution of integrated image points.

Consider that the reconstructed point we estimate is $(x, y, Z)$. Then, the intensity value of the corresponding image point for each image can be obtained according to the positional relation between the reconstruction point and the image sensor. Let $\left(\xi_{i}, \eta_{i}\right)$ and $I_{i}\left(\xi_{i}, \eta_{i}, z_{i}\right)$ denote the position coordinate and the intensity of the corresponding image point on the $i$-th image sensor, respectively. Here, $z_{i}=z_{1}+(i-1) \Delta z$ denotes the distance between the $i$-th image and the origin ( $z=0$ ). As shown in Fig. 3, we can see that $\square z=\square d \times \cos \theta$ and $\square x=\square d \times \sin \theta$, then we can obtain that

$$
\xi_{i}=\frac{-g}{Z-(i-1) \square z-g} y
$$

$$
\eta_{i}=\frac{-g}{Z-(i-1) \square z-g}\left[x+(i-1) \frac{\square x}{p}\right]
$$

where $p$ is the pixel size. From Eqs. (1) and (2), it is obvious that $\left(\xi_{i}, \eta_{i}\right)$ is varied according to $Z$. In details, we implemented two steps to obtain $I_{i}\left(\xi_{i}, \eta_{i}, z_{i}\right)$ by the recorded multi-perspective image set. First, we choose the first image as a template and shift the other images in column by the following:

$$
S=\eta_{i}-\eta_{1}=\frac{-g(i-1) \square x}{(Z-(i-1) \square z-g) p}
$$

Then the shifted images are normalized as following:

$$
M=\frac{M_{i}}{M_{1}}=-\frac{Z-(i-1) \square z-g}{Z-g}
$$


1 After $I_{i}\left(\xi_{i}, \eta_{i}, z_{i}\right)$ is calculated, we can use the statistical variance of the intensity values for all

2 image points to find whether the reconstruction point is a $3 \mathrm{D}$ object point or not.

3 We average the intensities of all the corresponding image points at the reconstruction point $4 \quad(x, y, z)$. It can be calculated as:

$$
m(x, y, Z)=\frac{1}{N} \sum_{i=1}^{N} I_{i}\left(\xi_{i}, \eta_{i}, z_{i}\right)
$$

6 Then, variance metric $D$ is defined as

$$
D(x, y, Z)=\frac{1}{N} \sum_{i=1}^{N}\left[I_{i}\left(\xi_{i}, \eta_{i}, z_{i}\right)-m(x, y, Z)\right]^{2}
$$

In Eq. (6), the variance metric $D$ is varied according to the $Z$ distance. Finally, 3D object point can be estimated by finding the local minimum of variance metric $D$ as the following

$$
\hat{z}(x, y)=\arg \min D(x, y, Z)
$$

When the local minimum variance metric is obtained, the intensity value of $3 \mathrm{D}$ object point can be represented as the mean value by the following:

$$
C(x, y)=\frac{1}{N} \sum_{i=1}^{N} I_{i}\left[\left(\frac{-g}{\hat{z}(x, y)-(i-1) \square z-g} y, \frac{-g}{\hat{z}(x, y)-(i-1) \square z-g}\left[x+\frac{\square x(i-1)}{p}\right], z_{i}\right)\right]
$$

The depth extraction process is repeated for all $x$ and $y$ to obtain the final depth map and the color image of $3 \mathrm{D}$ object.

\subsection{Elemental image array generation process}

Using the estimated final depth map and color image, we generate the elemental image array of the $3 \mathrm{D}$ object for 3D reconstruction based on the geometry of pixel mapping between 3D object points and the elemental images plane through the lenslet array (in fact, virtual pinhole array). As shown in Fig. 4, the rays emitted from 3D objet point $C(x, y)$ will pass through the center of each virtual pinhole and then recorded into the corresponding pixels $E\left(u_{k}, v_{k}\right)$ in the 
elemental image plane. The coordinates for the recorded points in each elemental image can be written as

$$
\begin{gathered}
u_{k}=P_{k, h}-\left(x-P_{k, h}\right) \frac{f}{\hat{z}(x, y)} \\
v_{k}=P_{k, v}-\left(y-P_{k, v}\right) \frac{f}{\hat{z}(x, y)}
\end{gathered}
$$

where $f$ is the focal length of lenslet and $P_{k, h}$ and $P_{k, v}$ are the horizontal and vertical position of the $k$-th pinhole, respectively. For all pixels, by iteration of the pixel mapping process, the final elemental image array can be obtained. Then, 3D images can be displayed in free space by displaying the elemental image array through the lenslet array in depth priority integral imaging system or reconstructed in 3D computational integral imaging system.

\section{Experiments and results}

Preliminary experiments are performed to demonstrate the proposed scheme. Figure 5 shows the experimental setup of the ODIS process. In this paper, three toy cars are used to compose a 3D scene, where the car1, car2 and car3 are located at $620 \mathrm{~mm}, 760 \mathrm{~mm}$ and $900 \mathrm{~mm}$ from the first image sensor plane, respectively. The multiple $2 \mathrm{D}$ images are recorded by moving single image sensor along the slanted axis $d$. The angle between the slanted axis and the optical axis is $45^{\circ}$. The imaging lens with a focal length $f=35 \mathrm{~mm}$ is used in this experiments. The camera is translated with $d=7 \mathrm{~mm}$ increments for a total of $K=41$ elemental images and a total displacement distance is $280 \mathrm{~mm}$. Fig. 5(b) and 5(c) show two recorded 2D images (1st image and 41th image), respectively.

To synthesize the elemental image array, the profilometry-based depth extraction process is performed to estimate the depth map and color image. The extracted color image and depth map are shown in Fig. 6. Their resolution is $1060(\mathrm{H}) \times 550(\mathrm{~V})$. Then, the extracted depth map and color image are used to synthesize the EIA for optical display and computational display as explained in Fig. 4. 


\subsection{Optical display}

For optical display, in or der to fit the display screen, we resize the extracted depth map and color image to $3731(\mathrm{H}) \times 2002(\mathrm{~V})$. To generate the new elemental image array, the left car is relocated at $\mathrm{z}=-12 \mathrm{~mm}$ (virtual image), the middle car is relocated at $\mathrm{z}=54 \mathrm{~mm}$ (real image) and the right car is relocated at $z=126 \mathrm{~mm}$. The elemental image array consists of $287(\mathrm{H}) \times 154(\mathrm{~V})$ elemental images shown in Fig. 7(a) and each elemental image has $13 \times 13$ pixels. To optically display the

3D scene, the lenslet array, which consists of $287(\mathrm{H}) \times 154(\mathrm{~V})$ lenslets whose pitch and focal length are $1.6056 \mathrm{~mm}$ and $8.028 \mathrm{~mm}$, is located in front of the display screen. Fig. 7(b)-(f) show the optically reconstructed 3D images.

\subsection{Computational reconstruction}

To further demonstrate the feasibility of the proposed method, we also implement the computational reconstruction using synthesized elemental image array. Figure 8(a) shows the synthesized elemental image array which has a resolution $3731(\mathrm{H}) \times 2002(\mathrm{~V})$. Fig. 8(b)-(d) shows that the left green car, the middle white caer and the right green care are reconstructed at $\mathrm{t} z=-12$ $\mathrm{mm}, \mathrm{z}=54 \mathrm{~mm}$ and $\mathrm{z}=126 \mathrm{~mm}$, respectively. The computational experimental results can also verify the correctness of the synthesized elemental image array. Additionally, to quantitatively evaluate the simulation results, the PNSR is employed as a measure of image quality. The PSNRs of the reconstructed object images of the Car1, Car2 and Car3 with the conventional and the proposed methods are $32.01 \mathrm{~dB}, 24.81 \mathrm{~dB}$ and $24.29 \mathrm{~dB}$, and $35.46 \mathrm{~dB}, 25.33 \mathrm{~dB}$ and $24.49 \mathrm{~dB}$, respectively. These results also confirmed that the object image reconstructed by using the proposed method achieve better visual quality than the conventional method.

\section{Conclusion}

In this paper, 3D integral imaging display system has been proposed using off-axially recorded multi-perspective 2D images. The statistical variance of image points from the $3 \mathrm{D}$ object is used to extract the depth and intensity information of the 3D scene. With the extracted depth map and 
color image, the elemental images are computationally synthesized based on ray mapping model between 3D space and elemental image plane. As a result, 3D images can be optically reconstructed by integrating the rays from the synthesized elemental images through the lenslet array. The experimental results show that the proposed method can display 3D images optically and computationally with the elemental image array synthesized from off-axially recorded multiperspective $2 \mathrm{D}$ images.

\section{Acknowledgement}

This work was supported by the National Science Foundation of China (No.61405022) and the Fundamental Research Funds for the Central Universities (DUT15RC(3)046) and the Basic Science Research Program through the National Research Foundation of Korea (NRF) funded by the Ministry of Education (NRF-2013R1A1A2057549).

\section{References}

1. G Lippmann, "La photographie integrale," C. R. Acad. Sci. 146, 446-451 (1908).

2. D.-H. Shin, S.-H. Lee and E.-S. Kim, "Optical display of true 3D objects in depth-priority integral imaging using an active sensor," Opt. Commun. 275, 330-334 (2007).

3. J.-H. Park, K. Hong, and B. Lee, "Recent progress in three-dimensional information processing based on integral imaging," Appl. Opt. 48(34), H77-H94 (2009).

4. S. Yeom, Y.-H. Woo, and W.-W. Beak, "Distance extraction by means of photon-counting passive sensing combined with integral imaging," J. Opt. Soc. Korea 15, 357-361(2011).

5. G Li, K. -C. Kwon, G. -H. Shin, J. -S. Jeong, K. -H. Yoo, and N. Kim, “Simplified integral imaging pickup method for real objects using a depth camera," J. Opt. Soc. Korea 16, 381385 (2012).

6. Y. Piao, D. Shin, E. -S. Kim, "Robust image encryption by combined use of integral imaging and pixel scrambling techniques," Opt. and Lasers in Engineering 47, 1273-1281 (2009).

7. X. -W. Li, S. J. Cho, and S. T. Kim, "Combined use of BP neural network and 
computational integral imaging reconstruction for optical multiple-image security," Opt. Commun. 315, 147-158 (2014).

8. D. -C. Hwang, D. -H. Shin, and E. -S. Kim. "A novel three-dimensional digital watermarking scheme basing on integral imaging, "Opt. Commun. 277, 40-49 (2007).

9. X. -W. Li, and I. -K. Lee, "Robust copyright protection using multiple ownership watermarks," Opt. Express 23, 3035-3046 (2015).

10. Y. Piao, M. Zhang, D. Shin, and H. Yoo, "Three-dimensional imaging and visualization using off-axially distributed image sensing, "Opt. Lett. 38(16), 3162-3164 (2013).

11. M. Zhang, Y. Piao, N.-W. Kim, and E. -S. Kim, "Distortion-free wide-angle 3D imaging and visualization using off-axially distributed image sensing," Opt. Lett. 39(14), 4212-4214 (2014).

12. M. Zhang, Y. Piao, and M. Cho, "Monte Carlo analysis of voxel resolution of off-axially distributed image sensing," Opt. Commun.363, 176-180 (2016).

13. R. Schulein, M. DaneshPanah, and B. Javidi, "3D imaging with axially distributed sensing," Opt. Lett. 34(13), 2012-2014 (2009).

14. D. Shin and B. Javidi, "Visualization of 3D objects in scattering medium using axially distributed sensing," J. Disp. Technol. 8(6), 317-320 (2012).

15. H. Yoo, "Axially moving a lenslet array for high-resolution 3D images in computational integral imaging," Opt. Express 21(7), 8873-8878 (2013).

16. M. Cho and D. Shin, "3D integral imaging display using axially recorded multiple images," J. Opt. Soc. Korea 17(5), 410-414 (2013). 


\section{$1 \quad$ Figure Captions}

Fig. 1. Block diagram of the proposed 3D display system.

Fig. 2. Pickup multi-perspective images using ODIS.

Fig. 3. Ray model for off-axially recording of 3D object point.

Fig. 4. Ray mapping model for elemental image array generation.

Fig. 5. (a) Experimental pickup setup, (b) 1st recorded 2D image, (c) 41th recorded 2D image.

Fig. 6. (a) Extracted color image, (b) Extracted depth map.

Fig. 7. (a) Generated elemental image array. (b)-(f) are experimental results, (b) Center view, 


\section{ODIS Profilometry-based Computational 3D integral}

pickup $\Rightarrow$ depth extraction EIA synthesis imaging display

4

Figure 1.

6 


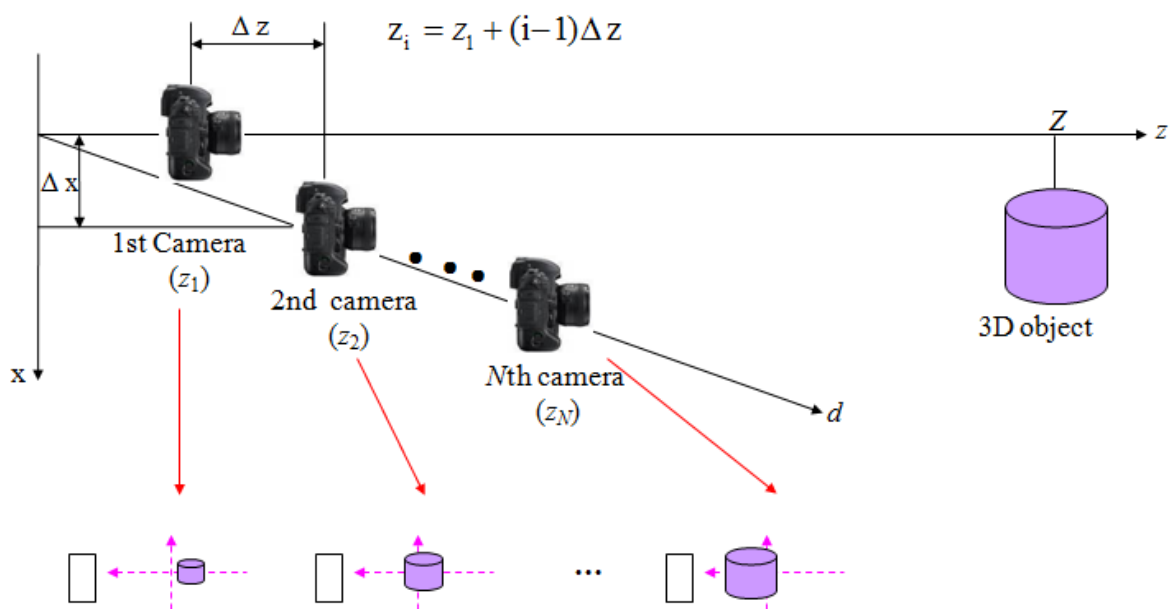

Off-axially recorded multiple images (Elemental image set)

Figure 2. 


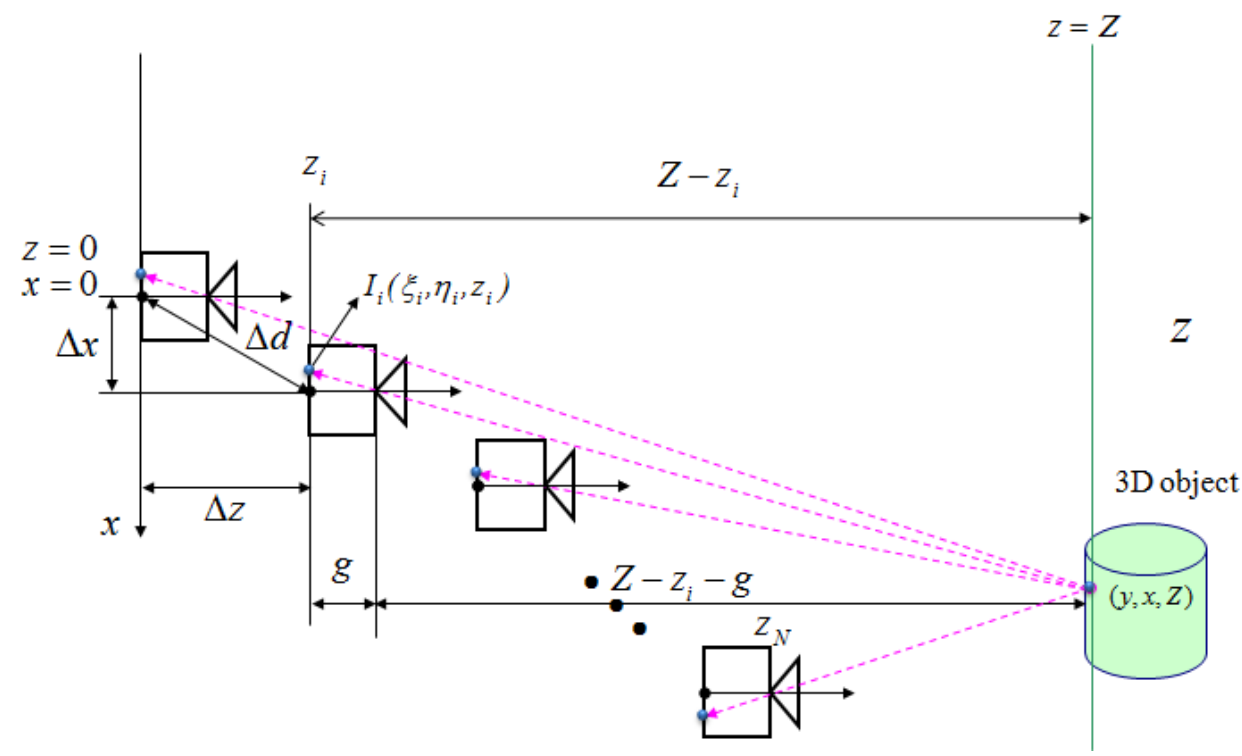

4

Figure 3.

6

7

8

9

10

11

12

13

14

15

16

17

18 


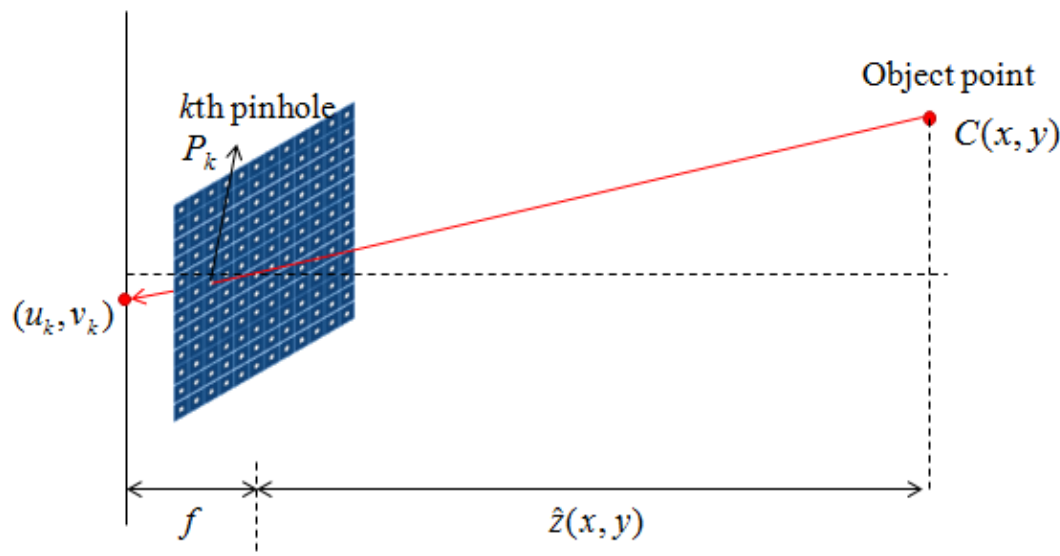

4

Figure 4

6

7

8

9 


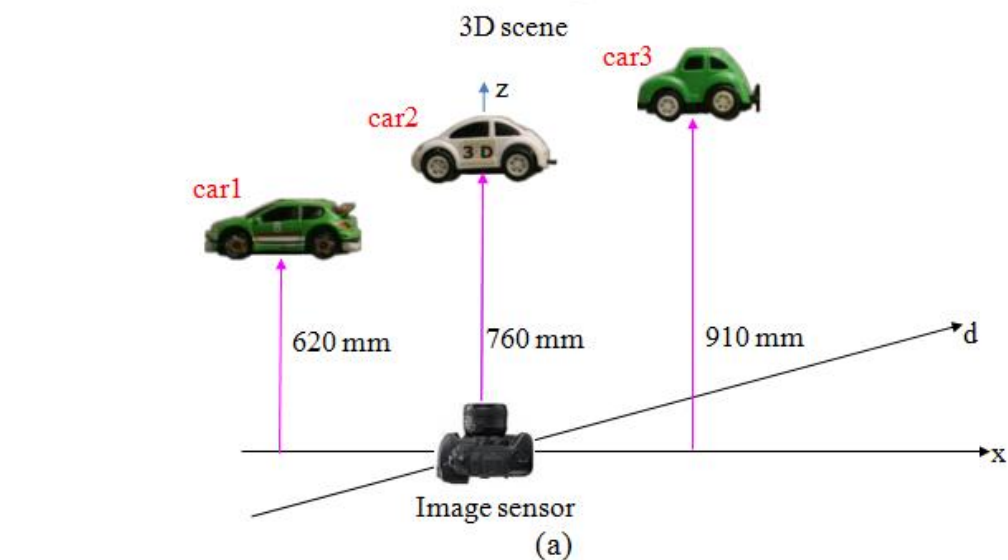

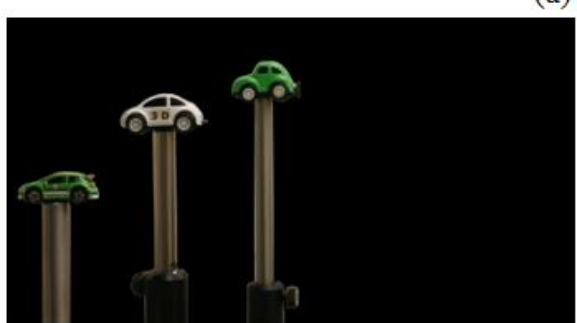

(b)

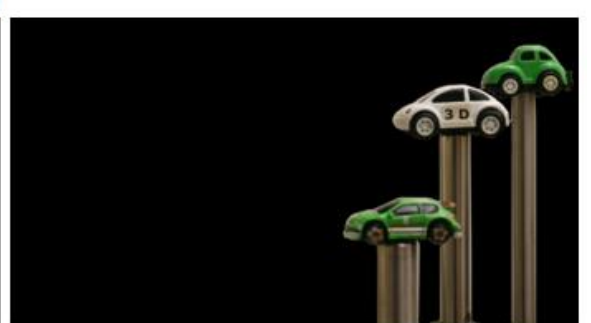

(c)
3

4

5

6

Figure 5 


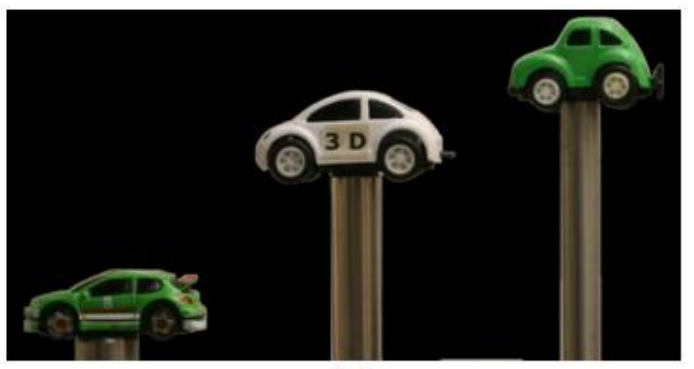

(a)

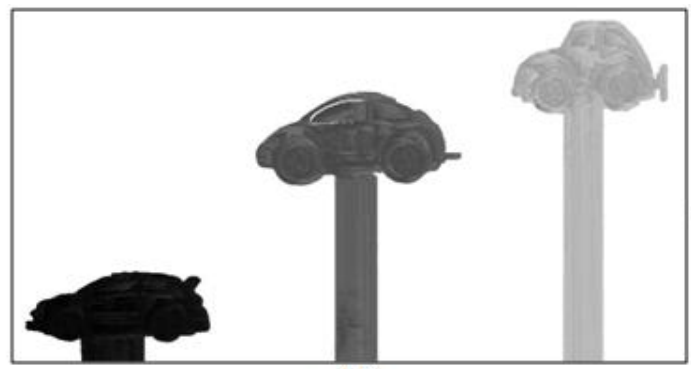

(b)

Figure 6

6

7

8

9 


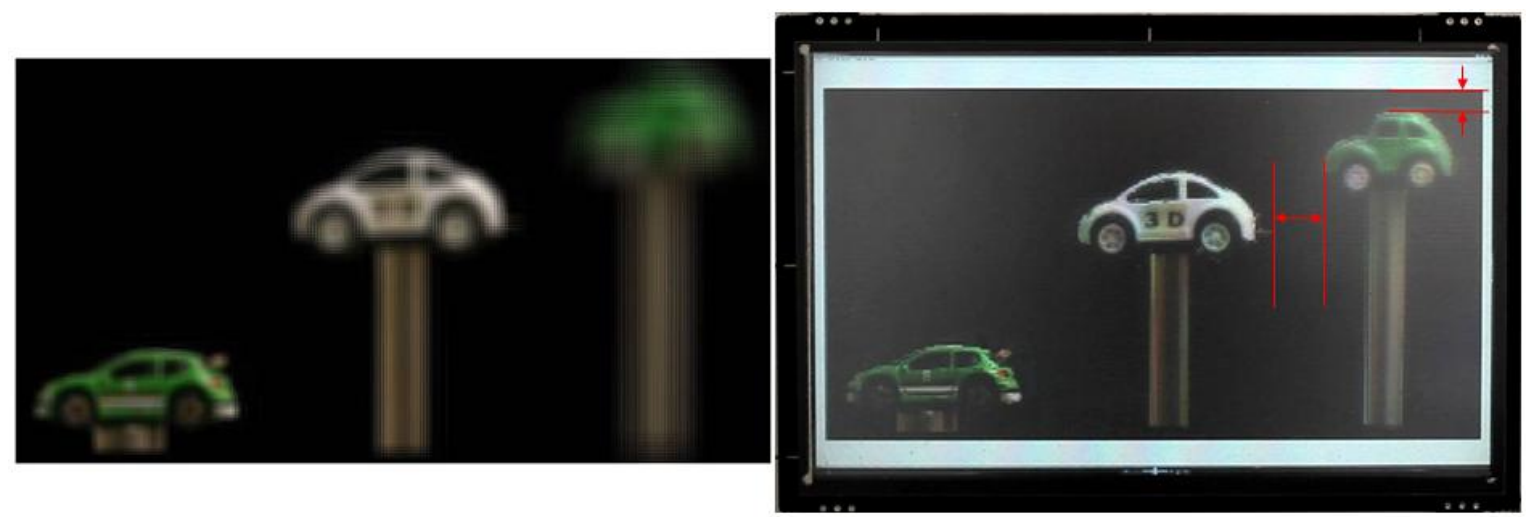

(a)

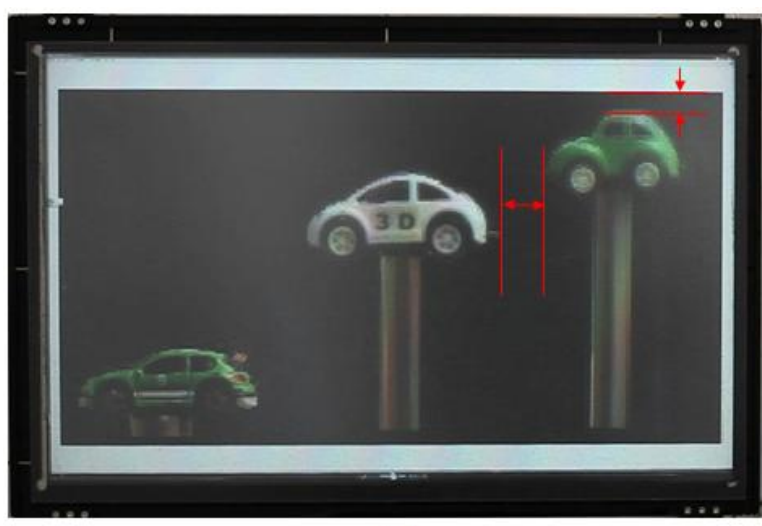

(c)

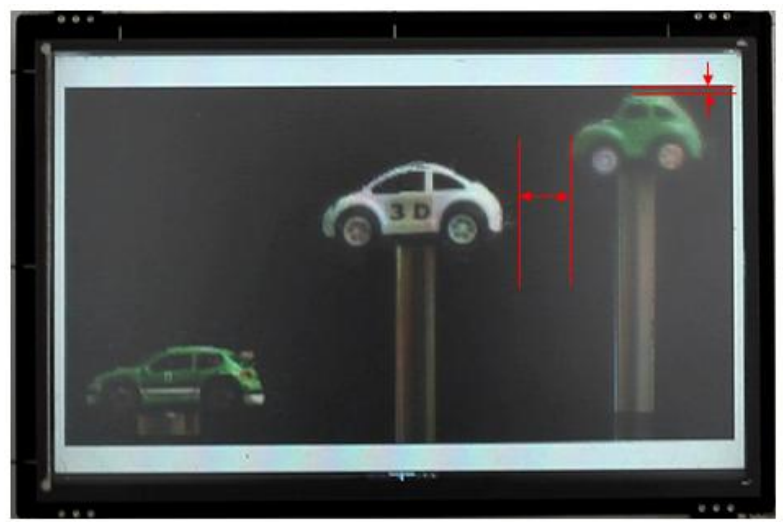

(e) (b)

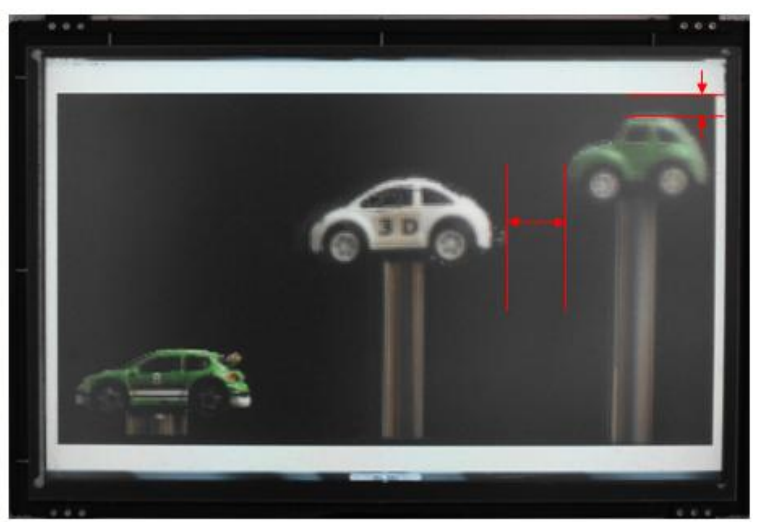

(d)

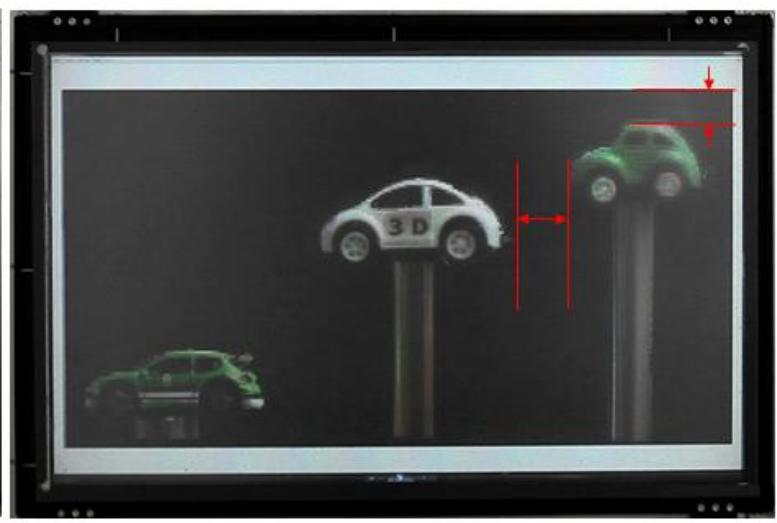

(f)

Figure 7 


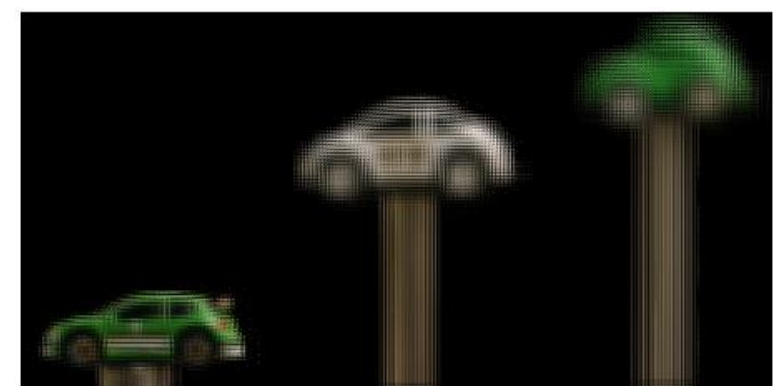

(a)

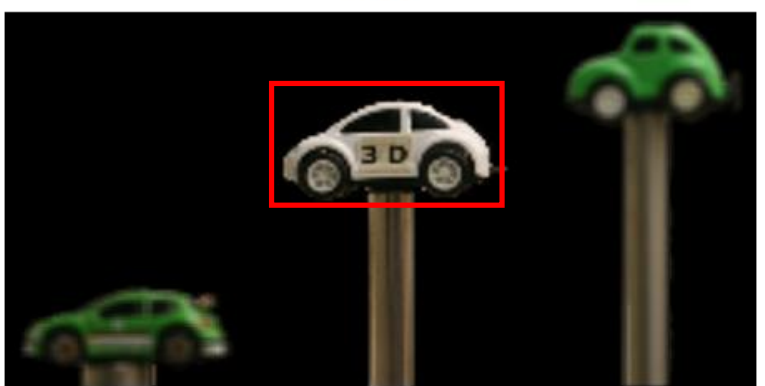

(c)

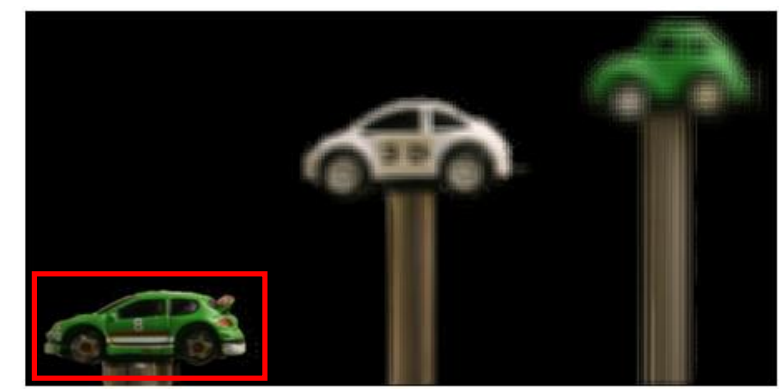

(b)

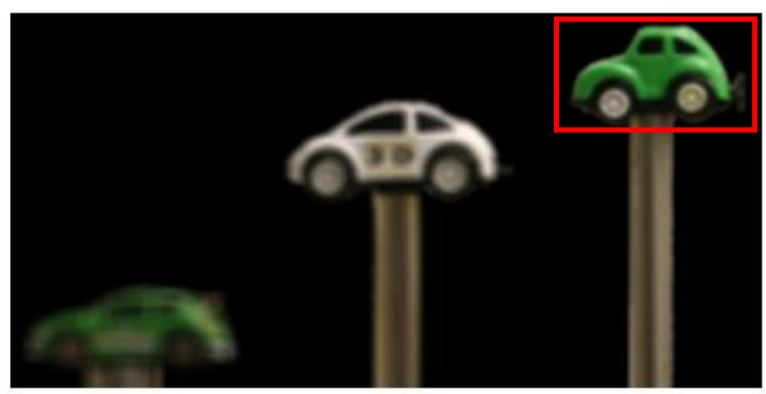

(d)

3

Figure 8 